\title{
ON SOME STOCHASTIC PARABOLIC DIFFERENTIAL EQUATIONS IN A HILBERT SPACE
}

\author{
KHAIRIA EL-SAID EL-NADI
}

Received 12 March 2004 and in revised form 29 July 2004

We consider some stochastic difference partial differential equations of the form $d u(x, t, c)$ $=L(x, t, D) u(x, t, c) d t+M(x, t, D) u(x, t-a, c) d w(t)$, where $L(x, t, D)$ is a linear uniformly elliptic partial differential operator of the second order, $M(x, t, D)$ is a linear partial differential operator of the first order, and $w(t)$ is a Weiner process. The existence and uniqueness of the solution of suitable mixed problems are studied for the considered equation. Some properties are also studied. A more general stochastic problem is considered in a Hilbert space and the results concerning stochastic partial differential equations are obtained as applications.

\section{Introduction}

Consider the stochastic linear system

$$
d u(t, c)=A u(t, c) d t+\sum_{i=1}^{n} \sum_{j=1}^{k} b_{i j}\left(B_{i} u\left(t-c_{j}, c\right)\right) d w_{i j}(t)
$$

where $A$ is a linear closed operator generating the strongly continuous semigroup $Q(t)$ on a separable Hilbert space $H$, and $w_{i j}$ are mutually independent Wiener processes on a separable Hilbert space $K$ with covariance operators $W_{i j}$, positive nuclear operators in the space $L(K, K)$ of continuous linear mapping of $K$ into itself.

It is assumed that $A$ is defined on $S_{1} \subset H$ into $H$ and $S_{1}$ is dense in $H$ (see [4]).

It is assumed also that $B_{1}, \ldots, B_{n}$ are linear closed operators defined on $S_{2} \supset S_{1}, S_{2} \subset H$, and with values in $H$.

$b_{i j}(\cdot)$ are elements of $L(H, L(K, H))$, (see $\left.[1,2,4]\right)$. We will study the existence and uniqueness of mild solutions, in other words, the existence and uniqueness of a solution of the equation

$$
u(t, c)=Q(t) u_{0}+\sum_{i=1}^{n} \sum_{j=1}^{k} \int_{0}^{t} Q(t-\theta) b_{i j}\left(B_{i} u\left(\theta-c_{j}, c\right)\right) d w_{i j}(\theta)
$$


We write $\|u\|$ for the Hilbert space norm of $u$, and $\left\|b_{i j}(u)\right\|$ for the norms of $b_{i j}(u)$ in $L(H, L(K, H))$. We write $\operatorname{tr} W_{i j}$ for the trace of $W_{i j}$. The processes $w_{i j}(t)$ are defined on a probability space $(\Omega, F, P)$. We denote by $E[u]$ the expectation of $u$. We suppose that the initial condition $u_{0}$ is independent of

$$
w_{i j}(t)-w_{i j}(s), \quad t \geq s>0
$$

for all $i=1, \ldots, n, j=1, \ldots, k$.

We suppose also that

$$
E\left[\left\|u_{0}\right\|^{2}\right]<\infty
$$

and that there is a number $\gamma \in(0,1)$ such that

$$
\left\|Q(t) b_{i j}\left(B_{i} f\right)\right\| \leq \frac{\alpha}{t^{\gamma / 2}}\|f\|,
$$

where $\alpha$ is a positive constant and $f \in S_{2}$.

For $f \in H$, we suppose that

$$
\left\|B Q(t) b_{i j}(f)\right\| \leq \frac{\alpha}{t^{\gamma / 2}}\|f\| .
$$

In Section 2, we will study the uniqueness and existence of $w_{i j}$ adapted solution $u(t, c)$ of $(1.2)$ in the space $C\left(0, T ; L_{2}(\Omega, H)\right)$, where $C([0, T], \Lambda)$ denotes the space of continuous functions mapping $[0, T]$ into $\Lambda \subset K$.

In Section 3, we study a mixed problem (initial and boundary value problem) of some stochastic difference partial differential equations.

\section{Uniqueness and existence of mild solutions}

Let $u(t, c)$ satisfy the condition

$$
u(t, c)=F(t), \quad-T_{0}<t<0,
$$

where $F$ is a given function in the space $C\left(\left[-T_{0}, 0\right], L_{2}(\Omega, H) \cap S_{2}\right)$.

We assume that

$$
F(0)=u_{0}
$$

We prove now the following theorem.

Theorem 2.1. Let $u \in C\left([0, T], L_{2}(\Omega, H)\right) \cap S_{2}$ be the solution of (1.2). If $F(t)=0$ on $\left[-T_{0}, 0\right]$, then $u(t)=0$ for all $t \geq 0$.

Proof. The solution of the above equation can be written in the form

$$
u(t, c)=\sum_{i=1}^{n} \sum_{j=1}^{k} \int_{\gamma_{j}(t)}^{t} Q(t-\theta) b_{i j}\left(B_{i} u\left(\theta-c_{j}, c\right)\right) d w_{i j}(\theta),
$$


where

$$
\gamma_{j}(t)= \begin{cases}t, & t \leq c_{j} \\ c_{j}, & t>c_{j}\end{cases}
$$

Thus,

$$
E\left[\|u(t, c)\|^{2}\right] \leq \sum_{i=1}^{n} \sum_{j=1}^{k} \operatorname{tr} W_{i j} \int_{\gamma_{j}(t)}^{t} \frac{\alpha^{2}}{(t-\theta)^{\gamma}} E\left[\left\|u\left(\theta-c_{j}, c\right)\right\|^{2}\right] d \theta
$$

So, there is a positive constant $\lambda$ such that

$$
E\left[\|u(t, c)\|^{2}\right] \leq \frac{\lambda M t^{1-\gamma}}{1-\gamma}
$$

where

$$
M=\sup _{\theta, c} E\|u(\theta, c)\|^{2}
$$

It is easy to see that

$$
E\left[\|u(t, c)\|^{2}\right] \leq \frac{1}{1-\gamma} t^{2(1-\gamma)} \lambda^{2} M \beta(1-\gamma, 2-\gamma),
$$

where $\beta(m, n)$ is the $\beta$ function. Now for every $r=1,2, \ldots$, we can prove that

$$
E\left[\|u(t, c)\|^{2}\right] \leq \frac{\lambda^{r} M t^{r(1-\gamma)}(\Gamma(1-\gamma))^{r}}{\Gamma(r(1-\gamma))+1},
$$

where $\Gamma$ is the gamma function.

Taking the limit as $r \rightarrow \infty$, we get the required result.

Now to prove the existence of solutions, we suppose that

$$
B_{1}=B_{2}=\cdots=B_{k}=B
$$

and $u_{0} \in S_{2}$.

Theorem 2.2. There exists a unique mild solution $u \in C\left([0, T], L_{2}(\Omega, H)\right) \cap S_{2}$ of (1.2).

Proof. We apply the method of successive approximation. To do this, we set

$$
\begin{aligned}
u_{r+1}(t, c)= & Q(t) u_{0}+\sum_{i=1}^{n} \sum_{j=1}^{k} \int_{0}^{\gamma_{j}(t)} Q(t-\theta) b_{i j}\left(B F\left(\theta-c_{j}\right)\right) d w_{i j}(\theta) \\
& +\sum_{i=1}^{n} \sum_{j=1}^{k} \int_{\gamma_{j}(t)}^{t} Q(t-\theta) b_{i j}\left(B u_{r}\left(\theta-c_{j}, c\right)\right) d w_{i j}(\theta) .
\end{aligned}
$$


Thus,

$$
\begin{aligned}
v_{r+1}(t, c)= & B Q(t) u_{0}+\sum_{i} \sum_{j} \int_{0}^{\gamma_{j}(t)} B Q(t-\theta) b_{i j}\left(B F\left(\theta-c_{j}\right)\right) d w_{i j}(\theta) \\
& +\sum_{i} \sum_{j} \int_{\gamma_{j}(t)}^{t} B Q(t-\theta) b_{i j}\left(v_{r}\left(\theta-c_{j}, c\right)\right) d w_{i j}(\theta),
\end{aligned}
$$

where

$$
v_{r}(t, c)=B u_{r}(t, c)
$$

The zero approximation is taken to be zero.

It is easy to see that

$$
E\left[\left\|v_{1}(t, c)\right\|^{2}\right] \leq E\left[\left\|B Q(t) u_{0}\right\|^{2}\right]+\sum_{i=1}^{n} \sum_{j=1}^{k} \operatorname{tr} W_{i j} \int_{0}^{\gamma_{j}(t)} \frac{\alpha^{2}}{(t-\theta)^{\gamma}}\left\|b_{i j}\right\|^{2} E\left[\left\|B F\left(\theta-c_{j}\right)\right\|^{2}\right] d \theta .
$$

Using the method of Theorem 2.1, we can prove that there exists a positive number $\lambda$ such that

$$
E\left[\left\|v_{r+1}(t, c)-v_{r}(t, c)\right\|^{2}\right] \leq \frac{\lambda^{r} t^{r(1-\gamma)}(\Gamma(1-\gamma))^{r}}{\Gamma(r(1-\gamma)+1)}
$$

Since $v$ can be written in the form

$$
v(t, c)=\sum_{r=0}^{\infty}\left[v_{r+1}(t, c)-v_{r}(t, c)\right]
$$

it follows that

$$
E\left[\|v(t, c)\|^{2}\right] \leq \sum_{r} \frac{1}{r^{2}(1-\gamma)^{2}} \sum_{r} r^{2}(1-\gamma)^{2} E\left[\left\|v_{r+1}(t, c)-v_{r}(t, c)\right\|^{2}\right] .
$$

So $v$ represents the solution of the equation

$$
\begin{aligned}
v(t, c)= & B Q(t) u_{0}+\sum_{i} \sum_{j} \int_{0}^{\gamma_{j}(t)} B Q(t-\theta) b_{i j}\left(B F\left(\theta-c_{j}\right)\right) d w_{i j}(\theta) \\
& +\sum_{i} \sum_{j} \int_{\gamma_{j}(t)}^{t} B Q(t-\theta) b_{i j}\left(v\left(\theta-c_{j}, c\right)\right) d w_{i j}(\theta) .
\end{aligned}
$$

Using (1.2) and (2.18), we deduce the existence of the solution of (1.2) in the space

$$
C\left(0, T ; L_{2}(\Omega, H)\right) \cap S_{2} \text {. }
$$

The uniqueness of this solution follows from Theorem 2.1. 
We give now conditions for the second moment of $u(t, c)$ to decay exponentially. To state the third theorem, we need the following conditions.

$C_{1}$ : there are positive numbers $\alpha$ and $\mu$ such that

$$
\|Q(t)\| \leq \alpha e^{-\mu t}, \quad t>0 .
$$

This exponential stability of the semigroup is equivalent to the requirement that for all $\lambda>-\mu$,

$$
\left\|(\lambda I+A)^{-1}\right\| \leq \alpha(\lambda+\mu)^{-1} .
$$

$C_{2}:\|B Q(t) f\| \leq\left(\alpha / t^{\gamma / 2}\right) e^{-\mu t}\|f\|, t>0$.

$C_{3}:\|Q(t) B f\| \leq\left(\alpha / t^{\gamma / 2}\right) e^{-\mu t}\|f\|, t>0$.

Theorem 2.3. Assume conditions $C_{1}, C_{2}$, and $C_{3}$ then for sufficiently large $\mu$, constants a and $b$ can be found such that

$$
E\left[\|u(t, c)\|^{2}\right] \leq a E\left[\left\|u_{0}\right\|^{2}\right] e^{-b t}, \quad a>0, b>0
$$

Proof. Using conditions $C_{1}, C_{2}$, and $C_{3}$, and (1.2), we get

$$
h(t, c) \leq \lambda_{1}+\lambda_{2} \sum_{j=1}^{k} \int_{0}^{t} \frac{h\left(\theta-c_{j}, c\right)}{(t-\theta)^{\gamma}} d \theta,
$$

where $\lambda_{1}=\alpha^{2} E\left[\left\|u_{0}\right\|^{2}\right], \lambda_{2}>\alpha^{2} \operatorname{tr} W_{i j}, \lambda_{2}$ is a positive constant, and $h(t, c)=e^{2 \mu t} E[\| u(t$, c) $\left.\|^{2}\right]$.

Let $\left\{h_{r}\right\}$ be a sequence of functions such that

$$
h_{r+1}(t, c) \leq \lambda_{1}+\lambda_{2} \sum_{j=1}^{k} \int_{0}^{t} \frac{h_{r}\left(\theta-c_{j}, c\right)}{(t-\theta)^{\gamma}} d \theta,
$$

where the zero approximation is taken to be zero. As $r \rightarrow \infty$, we get

$$
h(t, c) \leq \lambda_{1} \sum_{r} \frac{\lambda_{2}^{r} t^{r(1-\gamma)}(\Gamma(1-\gamma))^{r}}{\Gamma(r(1-\gamma)+1)} .
$$

Using the properties of Mittag-Leffler function, we get

$$
h(t, c) \leq C_{1} \exp \left[t \lambda_{2}^{1 /(1-\gamma)}\left(\Gamma(1-\gamma)^{1 /(1-\gamma)}\right)\right]+\frac{C_{2}}{1+t^{(1-\gamma)}}
$$

where $C_{1}$ and $C_{2}$ are positive constants. Thus for a sufficiently large $\mu$, we get the required result. 
172 On some stochastic parabolic differential equations

\section{Stochastic parabolic differential equations}

Let $C^{m}(S)$ be the set of all continuous functions in $S$ together with all their $m$-partial derivatives. Denote by $C_{0}^{m}(S)$ the subset of $C^{m}(S)$ consisting of all functions which have a compact support. Let $W^{m}(S)$ be a Sobolev space. In other words, $W^{m}(S)$ is the complete space of $C^{m}(S)$ with respect to the norm

$$
\|f\|_{m}=\left[\sum_{|\alpha| \leq m} \int_{S}\left|D^{\alpha} f(x)\right|^{2} d x\right]
$$

where $x=\left(x_{1}, \ldots, x_{n}\right)$,

$$
D^{\alpha}=D_{1}^{\alpha_{1}} \cdots D_{n}^{\alpha_{n}}, \quad D_{j}=\frac{\partial}{\partial x_{j}}, \quad j=1, \ldots, n,|\alpha|=\alpha_{1}+\cdots+\alpha_{n}
$$

and $\alpha=\left(\alpha_{1}, \ldots, \alpha_{n}\right)$ is an $n$-dimensional multi-index. We denote by $W_{0}^{m}(S)$ the complete space of $C_{0}^{m}(S)$ with respect to the norm defined by (3.1).

Let $r_{b}$ be the cylinder; $r_{b}=(x, t): x \in S, 0<t<b, 0<b<\infty$, and let $\Gamma_{b}$ be the lateral boundary

$$
\Gamma_{b}=\{(x, t): x \in \partial S, 0<t<b\}
$$

We consider the parabolic stochastic partial differential equations

$$
\begin{aligned}
d u(x, t, c)= & \sum_{i, j=1}^{n} a_{i j}(x, t) \frac{\partial^{2} u(x, t, c)}{\partial x_{i} \partial x_{j}} d t \\
& +\sum_{i=1}^{n} \sum_{r=1}^{k}\left[b_{i r}(x, t) \frac{\partial}{\partial x_{i}}+b_{0 r}(x, t)\right] u\left(x, t-c_{r}, c\right) d w_{i r}(t),
\end{aligned}
$$

with the initial and boundary conditions

$$
\begin{gathered}
u(x, 0, c)=u_{0}(x), \\
\left.u(x, t, c)\right|_{\Gamma_{b}}=0 .
\end{gathered}
$$

It is assumed that

$$
\sum_{i, j=1}^{n} a_{i j}(x, t) \xi_{i} \xi_{j} \geq \delta \sum_{i=1}^{n} \xi_{i}^{2}
$$

where $\delta>0,(x, t) \in \bar{\Omega}_{b}$ and $\bar{\Omega}_{b}$ is the closure of $\Omega_{b}$, and $\Omega_{b}$ is an open bounded domain in the $n+1$ dimensional Euclidean space. It is assumed also that all the coefficients $a_{i j}$, $b_{i r}$, and $b_{o r}$ are continuous on $\bar{\Omega}_{b}$ and satisfy a uniform Hölder condition in $t \in[0, b]$.

The mixed problem (3.4), (3.5), (3.6) can be written in the abstract form

$$
d u(t, c)=A u(t, c) d t+\sum_{i=1}^{n} \sum_{r=1}^{k} b_{i r}\left(B_{i} u\left(t-c_{r}, c\right)\right) d w_{i r}(t)+\sum_{r} b_{o r} u\left(t-c_{r}, c\right) d w_{o r}(t)
$$


where $A$ is the operator with domain $G=W^{2}(S) \cap W_{0}^{1}(S)$ given by

$$
A u=\sum_{i, j=1}^{n} a_{i j}(x) \frac{\partial^{2}}{\partial x_{i} \partial x_{j}} u
$$

Let $L_{2}(S)$ be the space of all square integrable functions on $S$. The space $H=L_{2}(S)$ is a Hilbert space and $G$ is dense in $H$.

The operators $B_{1}, \ldots, B_{n}$ with domains $W^{1}(S) \cap W_{0}^{1}(S)$ are given by

$$
B_{i}=\frac{\partial}{\partial x_{i}}, \quad i=1, \ldots, n
$$

and $b_{i r}, b_{o r}$ are the continuous functions defined on $\bar{\Omega}_{b}$.

Since $A$ is uniformly elliptic on $\bar{\Omega}_{b}$, it follows that the semigroup $Q(t)$ exists with the properties (1.5) and (1.6) (see $[3,5,6])$.

Consequently, Theorems 2.1, 2.2, and 2.3 can be applied for the considered abstract mixed problem.

\section{Acknowledgment}

I would like to thank the referees and Professor Eugene Dshalalow for their careful reading of the manuscript and their valuable comments.

\section{References}

[1] R. F. Curtain, Stability of stochastic partial differential equations, J. Math. Anal. Appl. 79 (1981), no. 2, 352-369.

[2] L. Delbeke and P. Abry, Stochastic integral representation and properties of the wavelet coefficients of linear fractional stable motion, Stochastic Process. Appl. 86 (2000), no. 2, 177-182.

[3] F. Flandoli, Stochastic flows for nonlinear second-order parabolic SPDE, Ann. Probab. 24 (1996), no. 2, 547-558.

[4] U. G. Haussmann, Asymptotic stability of the linear Itô equation in infinite dimensions, J. Math. Anal. Appl. 65 (1978), no. 1, 219-235.

[5] N. V. Krylov and B. L. Rozovskii, Itô equations in Banach spaces and strongly parabolic stochastic partial differential equations, Soviet Math. Dokl. 20 (1979), no. 2, 1267-1271 .

[6] V. H. de la Peña and S. J. Montgomery-Smith, Bounds on the tail probability of U-statistics and quadratic forms, Bull. Amer. Math. Soc. (N.S.) 31 (1994), no. 2, 223-227.

Khairia El-Said El-Nadi: Department of Mathematics, Faculty of Science, Alexandria University, P.O. Box 21511, Alexandria, Egypt

E-mail address: khairia_el_said@hotmail.com 


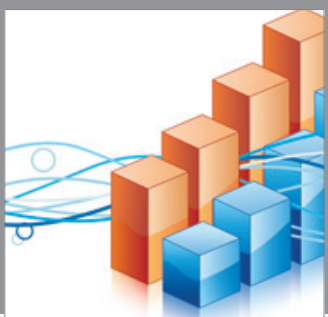

Advances in

Operations Research

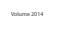

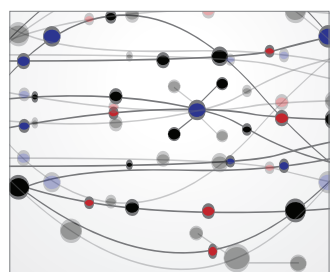

\section{The Scientific} World Journal
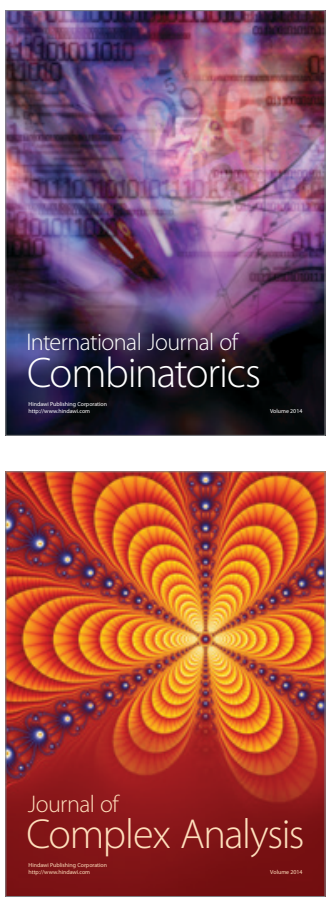

International Journal of

Mathematics and

Mathematical

Sciences
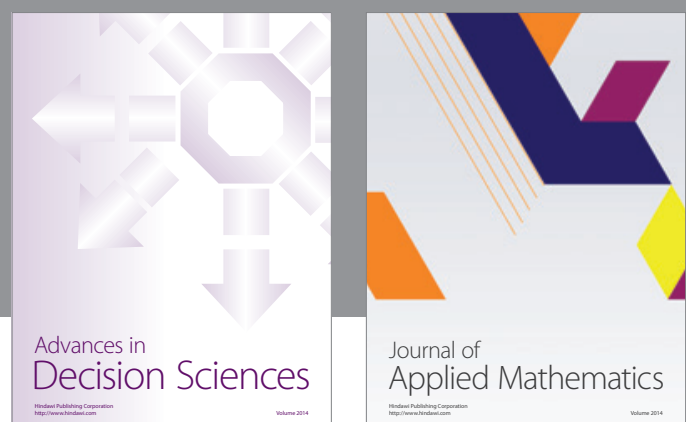

Journal of

Applied Mathematics
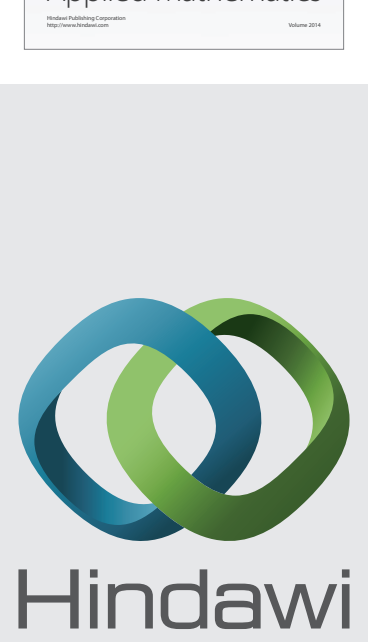

Submit your manuscripts at http://www.hindawi.com
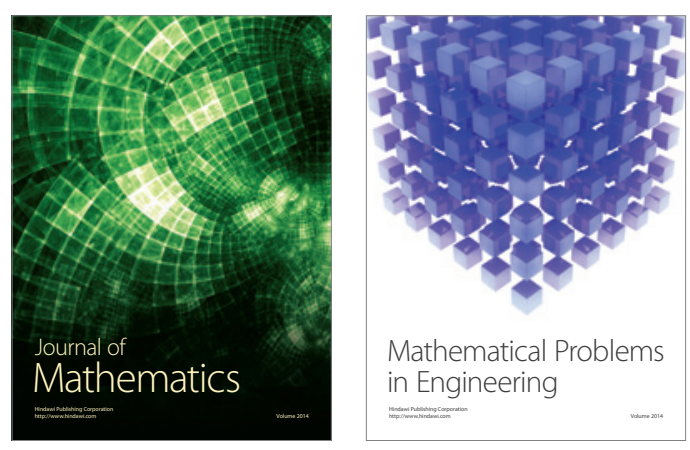

Mathematical Problems in Engineering
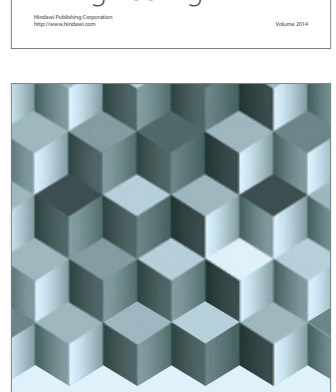

Journal of

Function Spaces
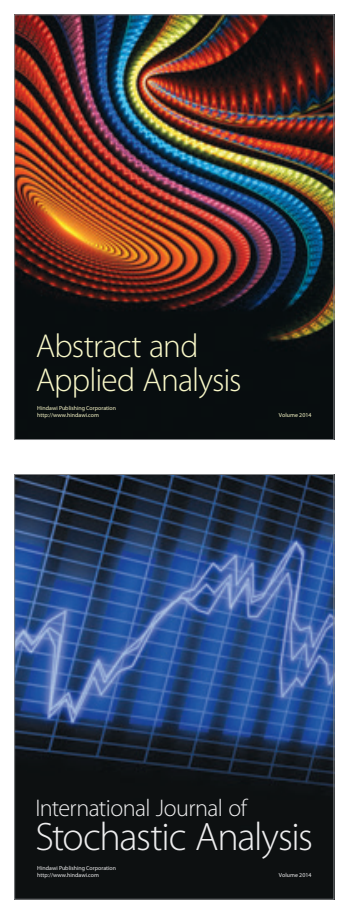

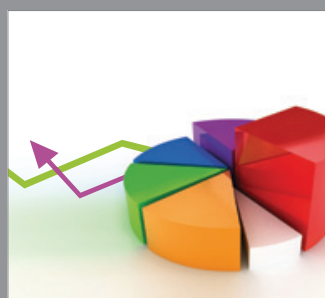

ournal of

Probability and Statistics

Promensencen
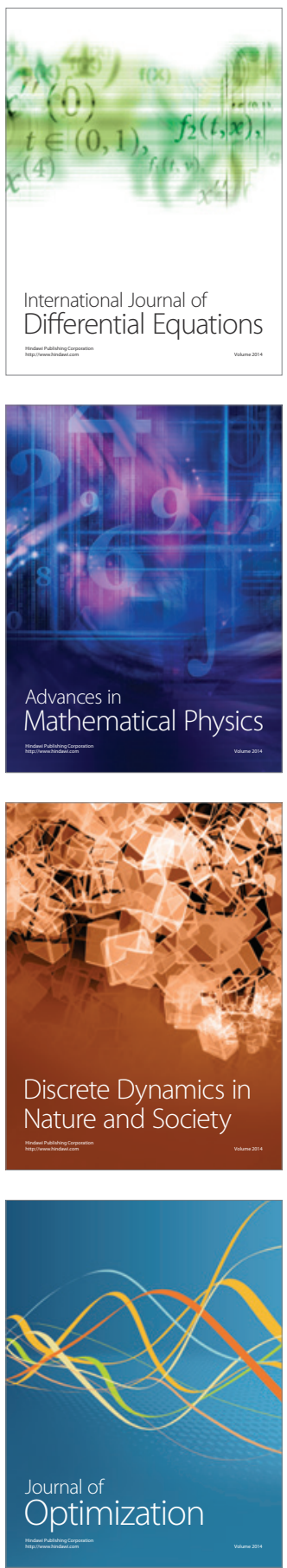